\title{
A CHILD WITH ACUTE MYELOID LEUKAEMIA COMPLICATED BY CALCANEAL OSTEOMYELITIS DUE TO MYCOBACTERIUM ABSCESSUS INFECTION AFTER INDUCTION CHEMOTHERAPY
}

\author{
Wilson Chan ${ }^{1}$, Pak Leung $\mathrm{Ho}^{2}$, Kelvin To ${ }^{2}$, Albert Lam ${ }^{1}$, Kenneth Ho${ }^{1}$, Tak Wing Lau ${ }^{1}$, \\ and Shau yin $\mathrm{Ha}^{1}$ \\ ${ }^{1}$ Queen Mary Hospital \\ ${ }^{2}$ University of Hong Kong Li Ka Shing Faculty of Medicine
}

September 21, 2020

\begin{abstract}
A 4-year old female with acute myeloid leukemia complicated with right calcaneal osteomyelitis due to Mycobacterium abscessus with subcutaneous abscesses extending to popliteal and groin regions after 2 courses of induction chemotherapy according to NOPHO-AML 2012 protocol. She required multiple operations and prolonged anti-mycobacterial therapy. High index of suspicion for mycobacterial infection is required for immunocompromised patients with prolonged fever or unusual presentation. Mycobacterial osteomyelitis is rare, difficult to diagnose and treat, and may necessitate prolonged interruption of anti-leukemic therapy. Multidisciplinary collaboration in patient management is crucial. Long term toxicity of antimicrobials with uncertain efficacy should not be overlooked.
\end{abstract}

\section{MAIN TEXT \\ INTRODUCTION}

Infection due to diverse organisms during induction chemotherapy for acute leukemia is often encountered due to immunosuppression. Severe bone and soft tissue infection due to non-tuberculous mycobacteria (NTM) in leukemic patient is possible but rare. Here we report a case of acute myeloid leukemia (AML) complicated with right calcaneal osteomyelitis due to Mycobacterium abscessus with subcutaneous abscesses extending to popliteal and groin regions requiring multiple operations and prolonged anti-mycobacterial therapy.

\section{CASE REPORT}

A 4-year old female with t $(8,21)$ AML and central nervous system involvement was treated with NOPHOAML 2012 protocol. She developed neutropenic fever 4 days after completed course 2 induction chemotherapy, which persisted for 1 week despite multiple antimicrobials and antifungals. She then complained of fluctuating right knee, hip and ankle pain over 4 weeks (Fig. 1). Clinical microbiologists and orthopedic surgeons were consulted. Subsequent magnetic resonance imaging (MRI) of right lower limb revealed multiple abscesses over right groin and popliteal region with calcaneal osteomyelitis and subcutaneous edema (Figs. 2A and 2B). Ultrasound-guided aspiration and biopsy yielded granulomas with surrounding suppurative inflammation(Fig. 2C). Smear of joint fluid was positive for acid-fast bacilli (AFB), while polymerase chain reaction (PCR) for Mycobacterium tuberculosis $(M . T B$ ) was negative. Child was covered with intravenous (IV) daptomycin, meropenem, levofloxacin and amikacin while awaiting identity and sensitivity testing of mycobacteria. Calcaneal bone excision and debridement of right ankle with incision and drainage (I\&D) 
were then performed followed by anti-mycobacterial chemotherapy with IV amikacin, oral clarithromycin, oral levofloxacin, and oral ethambutol (stopped after 1 week after revealing identity of NTM). Histology of excised os calcis bone confirmed osteomyelitis, and culture of bone and pus grew M. abscessus . Susceptibility testing of the $M$. abscessus isolate was determined by broth microdilution method and results were interpreted according to the Clinical and Laboratory Standards Institute. The isolate was susceptible to amikacin and trimethoprim-sulfamethoxazole, intermediate to cefoxitin, imipenem, linezolid, and resistant to ciprofloxacin, doxycycline and moxifloxacin. Antimicrobials were thus switched to oral trimethoprimsulfamethoxazole (with trimethoprim dosage $10 \mathrm{mg} / \mathrm{kg} /$ day), oral clarithromycin, IV imipenem-cilastatin (later switched to IV cefoxitin), IV amikacin and thrice weekly interferon-gamma (IFN- $\gamma$ ). In retrospect, the child might have sustained external injury to foot due to tight shoe according to mother.

Initial clinical response was noted but patient developed another abscess at right posterior thigh 12 days after the first operation as confirmed with PET-CT (Fig. 2D). Second operation was performed 3 weeks from first operation with I\&D of right groin and popliteal abscesses along with debridement of right ankle soft tissue achieved. Pus from posterior thigh grew M. abscessus. Low dose chemotherapy with oral thioguinine (6-TG) and IV with intrathecal cytarabine were resumed when fever subsided for 48 hours, and was given for 4 weeks, which led to worsening of infection necessitating third operation 4 weeks from second operation, involving sequestrectomy of right calcaneus and insertion of antibiotic-infused cement (vancomycin, amikacin and gentamycin). Child then developed drug-related rash and hepatitis with highest alanine aminotransferase and aspartate aminotransferase above 1500IU/L. Rash subsided and liver function improved after stopping all antimicrobials. Bedaquiline (100mg 3 days per week) and clofazimine (50mg 2 days per week) were introduced as salvage therapy for NTM infection 18 days from third operation. Antibiotic-infused cement was kept 7 months before removal and bone graft insertion. Peri-operatively patient was covered with 1week course of IV amikacin and imipenem-cilastatin. Intra-operative cultures did not yield any aerobic, anaerobic bacteria, fungi or AFB. Histology showed granulomatous inflammation with no evidence of AFB or fungi. Bone graft donor site and right calcaneal wounds healed well with stitches removed on postoperative day 11 and non-weight bearing for 6 weeks post-operatively. Bedaquiline and clofazimine were continued for 4 more weeks post-operatively then stopped. Total duration of usage was 8 months. Apart from mild prolongation of QTc from 450ms to 470ms during initiation of medication, she did not suffer from any adverse effects. Serial electrocardiograms and echocardiograms showed normal cardiac anatomy and function. Serial audiograms also reported normal hearing despite courses of prolonged amikacin. She was now 18 months from last operation. Limb function was preserved with no problem in walking and daily activities. For disease control, she had been followed up for 24 months since last chemotherapy given and remained in clinical and hematological remission with regular monitoring by 4 -weekly quantitative PCR (qPCR) and 8-weekly marrow exam. Minimal residual disease (MRD) was persistently negative $<0.1 \%$. No relapse so far for 2 years now.

\section{DISCUSSION}

\section{Prevalence and diagnostic challenges}

M. abscessus is an environmental rapidly-growing mycobacteria (RGM) pathogenic to humans $(1,2)$. Clinical manifestations include pneumonia $(3,4)$, lymphadenitis $(5,6)$, skin and soft tissue infections $(7)$, intravascular catheter related infections (8-11), systemic bacteremia and peritonitis (12). Cell-mediated immunity is the major protective immune response against intracellular bacteria $(13,14)$. Haemic malignancy and cytotoxic therapy impair phagocytic, cell-mediated and humoral immunity $(7,15)$ and thus predispose patients to NTM infections as in our patient. From a single-institution retrospective review in our locality, nearly all paediatric patients with NTM infection had an underlying congenital or acquired immunodeficiency (16).

\section{Dilemma between disease and infection control}

Outcome of paediatric AML is generally fair with an overall survival around $70 \%$ and a relapse rate of 30 to $40 \%$ after primary treatment. Infective complications post-induction is common but NTM infection is 
possible but rare, thus it had been postulated that our child reported might have an underlying congenital immune defect along the macrophage/interleukin-12/IFN- $\gamma$ pathway apart from acquired immunodeficiency due to haemic malignancy and cytotoxic therapy (16). Premature termination of chemotherapy without consolidation may increase relapse risk but further administration of high dose chemotherapy might result in uncontrolled disseminated NTM infection which could lead to limb amputation or even death. In view of high relapse risk without consolidation chemotherapy, the option of allogeneic hematopoietic stem cell transplantation (HSCT) had been explored and discussed. Potential benefits of HSCT include graftversus-leukemia effect which prevent disease relapse and reconstitution of healthy cellular immunity against mycobacterial infection but at the cost of ultra-high infection and mortality risk even with non-myeloablative reduced intensity conditioning or mini-transplant. After multidisciplinary discussion among paediatric oncologists, microbiologists, orthopedic surgeons together with parents and child, it was finally decided to control infection first and consider HSCT in case of disease relapse.

\section{Challenges in management of NTM infection}

Surgical debridement was the mainstay of treatment for bone and soft tissue infection (17) with prolonged anti-mycobacterial treatment in terms of at least 6 to 12 months (17-19). Drug-related toxicities and development of drug resistance may be encountered. As in our patient, antibiotics had to be stopped in view of drug-related rash and liver toxicity. Experience on use of bedaquiline and clofazimine as salvage therapy was limited without efficacy and safety profile for paediatric population documented in literature (20). Meticulous counselling of patient and parents before usage and close monitoring of potential side effects and drug interactions were crucial.

\section{CONCLUSION}

To conclude, high index of suspicion for mycobacterial infection is required for immunocompromised patients with prolonged fever or unusual presentation. Mycobacterial osteomyelitis is rare, difficult to diagnose and treat, and may necessitate prolonged interruption of anti-leukemic therapy. Multidisciplinary collaboration in patient management is crucial. Toxicity due to long-term use of antimicrobials should not be overlooked.

\section{ETHICS STATEMENT}

Informed consent had been properly obtained and documented for clinical photographs and radiological imaging used for demonstration in this manuscript. Identifiers on patient identity had been obscured prior to review,

\section{CONFLICT OF INTEREST}

The authors report no conflict of interest.

\section{ACKNOWLEDGEMENTS}

We would like to express our sincere thanks Dr PL Ho, Dr Kelvin To and Dr Sally Wong and their team from Department of Microbiology, Queen Mary Hospital / The University of Hong Kong and Dr YL Lam, Dr WY Ho, Dr TW Lau, Dr Noah So and their team from Department of Orthopedic and Traumatology, Queen Mary Hospital / Duchess of Kent Hospital for their expert opinion and patient management.

\section{LEGENDS}

Figure 1 Timeline of events from initiation of chemotherapy treatment to time of publication

\#1 Course 1 induction chemotherapy (MEC) with mitoxantrone $25 \mathrm{mg} / \mathrm{m}^{2}$, etoposide $750 \mathrm{mg} / \mathrm{m}^{2}$ and cytarabine $2800 \mathrm{mg} / \mathrm{m}^{2}$ administered.

\#2 Course 2 induction chemotherapy (ADxE) with cytarabine $1400 \mathrm{mg} / \mathrm{m}^{2}$, daunorubicin $180 \mathrm{mg} / \mathrm{m}^{2}$ and etoposide $450 \mathrm{mg} / \mathrm{m}^{2}$ administered.

1 Day 29 marrow confirmed remission status with $\mathrm{MRD}<0.1 \%$ 
2 Day 40 marrow before course 2 induction chemotherapy continued in remission with $\mathrm{MRD}<0.1 \%$

3 Bone marrow aspirate performed 2 weeks after completion of 1 course of low dose bridging chemotherapy (prior to consideration of starting consolidation chemotherapy) confirmed persistent remission status with $\mathrm{MRD}<0.1 \%$

4 Regular 4-weekly bone marrow aspirate confirmed remission with $\mathrm{MRD}<0.1 \%$

5 Regular 4-weekly bone marrow aspirate confirmed remission with $\mathrm{MRD}<0.1 \%$

1 MRI of right lower limb revealed bilateral shotty groin lymph nodes, largest $1.6 \mathrm{~cm}$ at right groin and $1 \mathrm{~cm}$ at right popliteal fossa, accompanied with subcutaneous edema over right upper medial and posterior distal thigh (Fig. 1A) . Neutrophil and monocyte counts were all along less than $1 \times 10^{9} / \mathrm{L}$ and $0.2 \times 10^{9} / \mathrm{L}$ respectively.

2 MRI of groins and right ankle showed abscess at right groin and lateral aspect of right ankle with underlying osteomyelitis of calcaneus(Fig 1B) .

3 MRI of bilateral lower limbs showing interval improvement in inflammatory changes over bilateral femur, tibia and right calcaneus

4 MRI of hips showing mild synovitis

$5 \mathrm{MRI}$ of right ankle showed minimal residual inflammatory changes at right calcaneus

Bx USG-guided aspiration and biopsy were performed, which yielded granulomas with surrounding suppurative inflammation (Fig. 1C) . A moderate number of AFB were highlighted on aspirate sample. Neutrophil and monocyte counts recovered.

1 PET-CT showed abscesses in right posterior thigh and popliteal fossa $\left(\mathrm{SUV}_{\max } 3.8\right)$, right calcaneal osteomyelitis $\left(\mathrm{SUV}_{\max } 9.4\right)$, as well as multiple hypermetabolic LN at right groin ( $\left.\mathrm{SUV}_{\max } 6.5\right)$ extending to right internal iliac LN (Fig. 1D) .

2 PET-CT showed worsening of right calcaneal osteomyelitis with increased bony lysis

3 PET-CT showed residual infective focus at right calcaneus with interval reduction in metabolic activity over right groin and right popliteal fossa.

4 PET-CT showed resolution of right groin lymphadenopathy and right popliteal fossa subcutaneous lesion, also decreased metabolic activity at right calcaneus.

$\S 1$ First operation on calcaneal bone excision and debridement of right ankle

$\S 2$ Second operation involving I\&D of right groin and popliteal abscesses along with debridement of right ankle soft tissue

$\S 3$ Third operation on sequestrectomy of right calcaneus and insertion of antibiotic-infused cement (vancomycin, amikacin and gentamycin)

$\S 4$ Fourth operation on removal of antibiotic-infused cement and bone graft insertion

Figure 2 (A) MRI of bilateral hips and thigh showing bilateral shotty groin LN accompanied with subcutaneous edema over right upper medial thigh and posterior distal thigh (B) MRI of right ankle showing T1 hypointense and $\mathrm{T} 2$ hyperintense signals at right talus, right distal tibia, right third proximal metatarsal, right distal fibula, right calcaneus and right posterior ankle. (C) Suppurative aspirate obtained by ultrasoundguided aspiration of right groin abscess (D) PET-CT 12 days after first operation showing abscesses in right posterior thigh and popliteal fossa $\left(\mathrm{SUV}_{\max } 3.8\right)$, right calcaneal osteomyelitis ( $\left.\mathrm{SUV}_{\max } 9.4\right)$, as well as multiple hypermetabolic $\mathrm{LN}$ at right groin $\left(\mathrm{SUV}_{\max } 6.5\right)$ extending to right internal iliac $\mathrm{LN}$

\section{REFERENCES}


1. Pozniak A, Bull T. Recently recognized mycobacteria of clinical significance. J Infect. 1999;38(3):157-61.

2. Jarzembowski JA, Young MB. Nontuberculous mycobacterial infections. Arch Pathol Lab Med. 2008;132(8):1333-41.

3. Martinez S, McAdams HP, Batchu CS. The many faces of pulmonary nontuberculous mycobacterial infection. Am J Roentgenol. 2007;189(1):177-86.

4. Wolfe J, Turenne C, Alfa M, Harding G, Thibert L, Kabani A. Mycobacterium branderi from both a hand infection and a case of pulmonary disease. J Clin Microbiol. 2000;38(10):3896-9.

5. Chetchotisakd P, Mootsikapun P, Anunnatsiri S, Jirarattanapochai K, Choonhakarn C, Chaiprasert A, et al. Disseminated infection due to rapidly growing mycobacteria in immunocompetent hosts presenting with chronic lymphadenopathy: a previously unrecognized clinical entity. J Clin Infect Dis. 2000;30(1):29-34.

6. Springer B, Kirschner P, Rost-Meyer G, Schröder K, Kroppenstedt R, Böttger E. Mycobacterium interjectum, a new species isolated from a patient with chronic lymphadenitis. J Clin Microbiol. 1993;31(12):3083-9.

7. Wallace Jr RJ, Brown BA, Onyi GO. Skin, soft tissue, and bone infections due to Mycobacterium chelonae chelonae: importance of prior corticosteroid therapy, frequency of disseminated infections, and resistance to oral antimicrobials other than clarithromycin. J Infect Dis. 1992;166(2):405-12.

8. Woo PC, Li JH, Tang WW, Yuen KY. Acupuncture mycobacteriosis. NEJM. 2001;345(11):842-3.

9. Raad II, Vartivarian S, Khan A, Bodey GP. Catheter-related infections caused by the Mycobacterium fortuitum complex: 15 cases and review. Rev Infect Dis. 1991;13(6):1120-5.

10. Schinsky MF, McNeil MM, Whitney AM, Steigerwalt AG, Lasker BA, Floyd MM, et al. Mycobacterium septicum sp. nov., a new rapidly growing species associated with catheter-related bacteraemia. Int J Syst Evol Microbiol. 2000;50(2):575-81.

11. Kiska DL, Turenne CY, Dubansky AS, Domachowske JB. First case report of catheter-related bacteremia due to "Mycobacterium lacticola". J Clin Microbiol. 2004;42(6):2855-7.

12. Vera G, Lew SQ. Mycobacterium fortuitum peritonitis in two patients receiving continuous ambulatory peritoneal dialysis. Am J Nephrol. 1999;19(5):586-9.

13. Griffith DE, Brown-Elliott BA, L. Benwill J, Wallace Jr RJ. Mycobacterium abscessus. "Pleased to meet you, hope you guess my name...". Ann Am Thorac Soc. 2015;12(3):436-9.

14. Tortoli E, Kohl TA, Brown-Elliott BA, Trovato A, Leão SC, Garcia MJ, et al. Amended description of Mycobacterium abscessus, Mycobacterium abscessus subsp. abscessus and Mycobacteriumabscessus subsp. bolletii and designation of Mycobacteriumabscessus subsp. massiliense comb. Int J Syst Evol Microbiol. Nov 2016;66(11):4471-9.

15. Snydman DR, Redelman-Sidi G, Sepkowitz KA. Rapidly growing mycobacteria infection in patients with cancer. J Clin Infect Dis. 2010;51(4):422-34.

16. Chan WYK LP, Chiang AKS, Cheuk KLD, Ha SY, Ho PL. Clinical Characteristics and Outcomes of Paediatric Non-tuberculous Mycobacterial Infection - Single institution retrospective review over 20 years and literature review. HK J Paediatr [In press].

17. Lindeboom JA, Kuijper EJ, Bruijnesteijn van Coppenraet ES, Lindeboom R, Prins JM. Surgical excision versus antibiotic treatment for nontuberculous mycobacterial cervicofacial lymphadenitis in children: a multicenter, randomized, controlled trial. J Clin Infect Dis. 2007;44(8):1057-64.

18. Sizaire V, Nackers F, Comte E, Portaels F. Mycobacterium ulcerans infection: control, diagnosis, and treatment. Lancet Infect Dis. 2006;6(5):288-96. 
19. Bermudez LE, Kolonoski P, Petrofsky M, Wu M, Inderlied CB, Young LS. Mefloquine, moxifloxacin, and ethambutol are a triple-drug alternative to macrolide-containing regimens for treatment of Mycobacterium avium disease. J Infect Dis. 2003;187(12):1977-80.

20. Harausz EP, Garcia-Prats AJ, Seddon JA, Schaaf HS, Hesseling AC, Achar J, et al. New and repurposed drugs for pediatric multidrug-resistant tuberculosis. Practice-based recommendations. Am J Respir Crit Care Med. 2017 May 15;195(10):1300-1310.
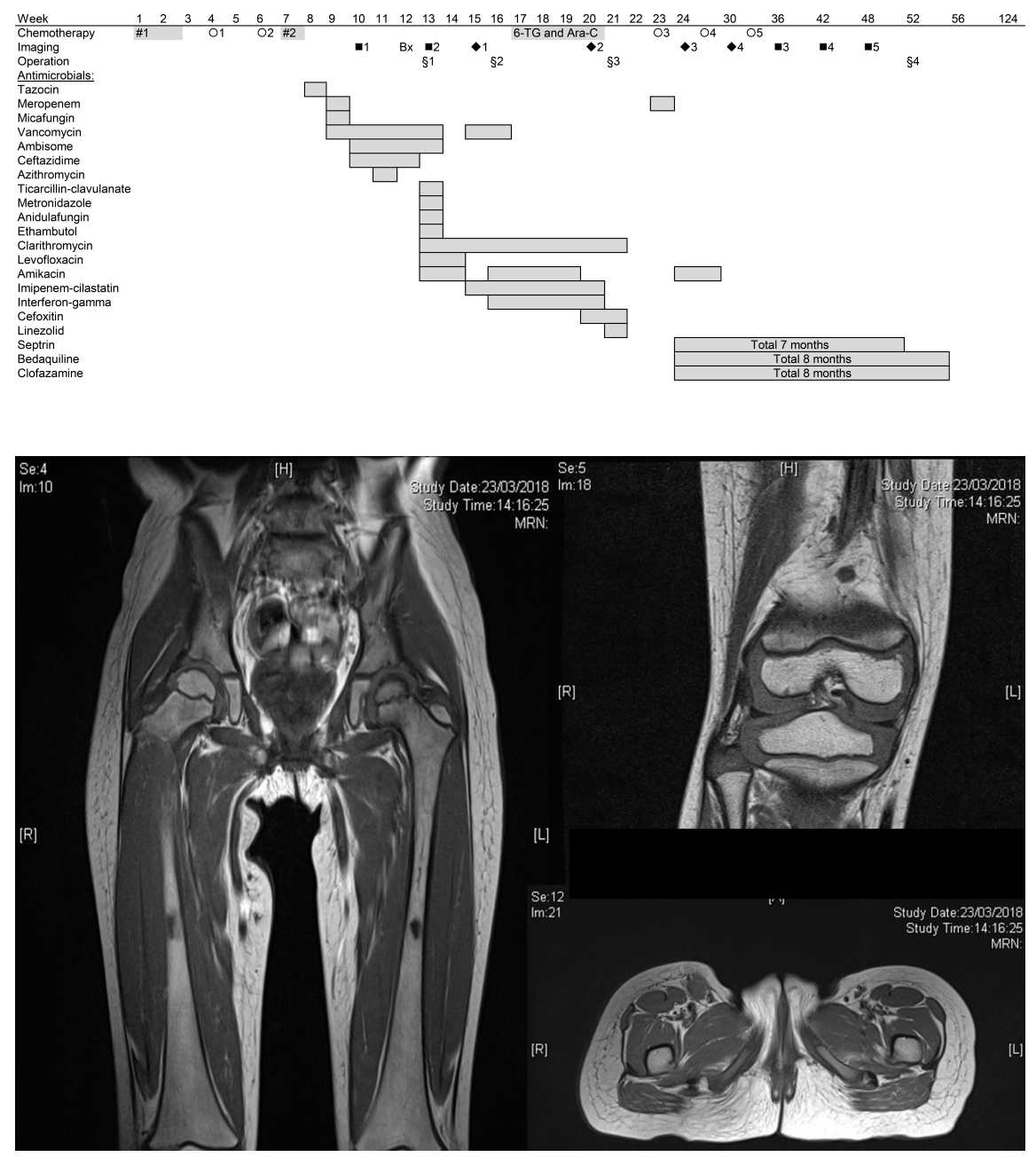

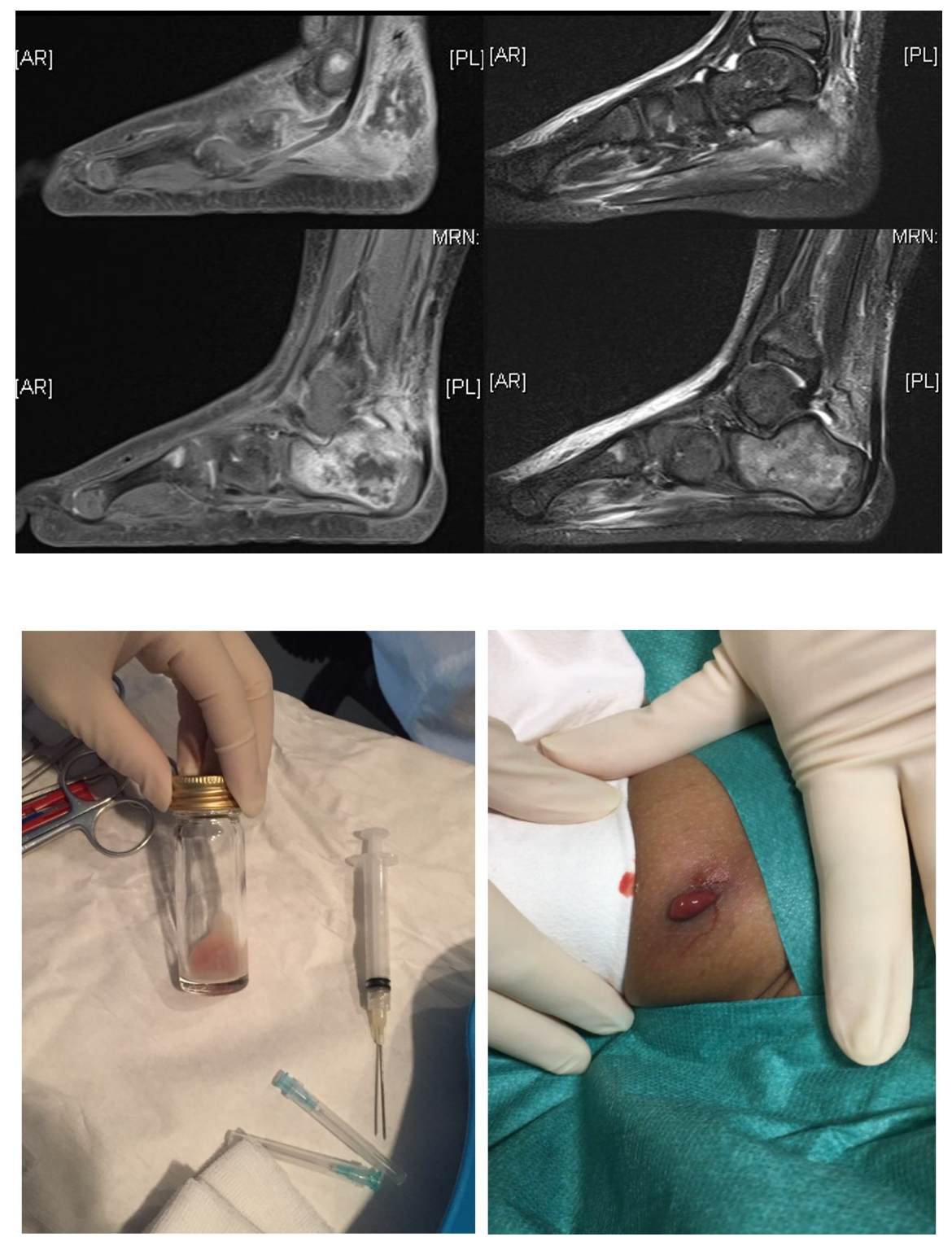


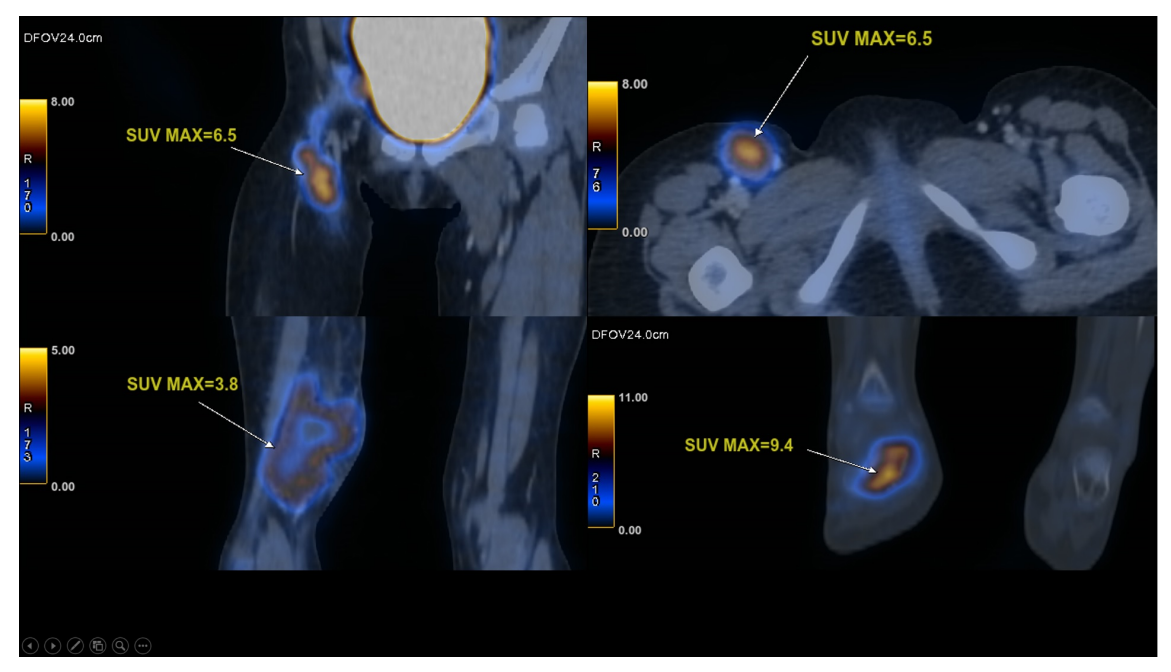

